\title{
Impact of patient and disease characteristics on therapeutic success during adalimumab treatment of patients with rheumatoid arthritis: data from a German noninterventional observational study
}

\author{
Stefan Kleinert • Hans-Peter Tony • Andreas Krause • Martin Feuchtenberger • \\ Siegfried Wassenberg • Constanze Richter • Ekkehard Röther • \\ Wolfgang Spieler • Holger Gnann • Bianca M. Wittig
}

Received: 8 February 2011 / Accepted: 10 July 2011 / Published online: 6 August 2011

(C) The Author(s) 2011. This article is published with open access at Springerlink.com

\begin{abstract}
The objective of this study was to use data from a noninterventional study to evaluate the effectiveness of adalimumab in rheumatoid arthritis (RA) patients during routine clinical practice and to explore the potential impact of patient and disease characteristics in response to adalimumab therapy. A total of 2,625 RA patients with specified data at baseline (prior to initiating adalimumab treatment) and 12 months entered this study between April 2003 and March 2009. We evaluated response to adalimumab therapy and conducted stepwise regression and subgroup analyses of factors influencing therapeutic
\end{abstract}

S. Kleinert $(\bowtie) \cdot$ H.-P. Tony $\cdot$ M. Feuchtenberger

Rheumatologie/Klinische Immunologie, Universitätsklinikum

Würzburg, Medizinische Klinik und Poliklinik II, Oberdürrbacherstr. 6, 97080 Würzburg, Germany

e-mail: Kleinert_S@klinik.uni-wuerzburg.de

A. Krause

Rheumatology Clinic, Immanuel Hospital, Berlin, Germany

S. Wassenberg

Ratingen Clinic, Ratingen, Germany

C. Richter

Rheumatology Medical Center, Stuttgart, Germany

E. Röther

Rheumatology Medical Center,

Villingen-Schwenningen, Germany

W. Spieler

Rheumatology Medical Center, Zerbst, Germany

H. Gnann

GKM Gesellschaft für Therapieforschung, Munich, Germany

B. M. Wittig

Abbott GmbH \& Co. KG, Wiesbaden, Germany response. During the 1-year adalimumab treatment period, disease activity decreased from a baseline mean disease activity score-28 joints (DAS28) of 5.9-3.9, while functional capacity improved from 59.0 to 68.4 Funktionsfragebogen Hannover $(\mathrm{FFbH})$ percentage points. In multivariate regression models, high baseline DAS28 was the strongest positive predictor for decrease in disease activity, and high baseline functional capacity was associated with reduced gains in functional capacity. Male gender was a positive predictor of therapeutic response for both disease activity and functional capacity, while older age and multiple previous biologics were associated with a reduced therapeutic response. Subset analyses provided further support for the impact of baseline DAS28, FFbH, and prior biologic therapy on therapeutic response during treatment. We conclude that treatment with adalimumab leads to decreased disease activity and improved function during routine clinical practice. Patients with high disease activity and low functional capacity are particularly benefitted by adalimumab therapy.

Keywords Adalimumab - Arthritis, rheumatoid · Treatment outcome $\cdot$ Regression analysis .

Antirheumatic agents

\section{Introduction}

Rheumatoid arthritis (RA) is a chronic inflammatory disease characterized by progressive joint destruction. As their joints deteriorate, patients experience pain and loss of function, often accompanied by decreased quality of life and increased mortality. Tumor necrosis factor- $\alpha$ (TNF- $\alpha)$ is an important cytokine that mediates inflammation in RA. Introduction of TNF- $\alpha$ inhibitors 10 years ago revolutionized 
RA treatment options and led to the development of further biologic disease-modifying antirheumatic drugs (DMARDs). Adalimumab, a human monoclonal antibody against TNF- $\alpha$, is approved for RA treatment in the United States (2002), the EU (2003), and other countries around the world. Several clinical trials have shown that adalimumab reduces the clinical symptoms of RA and decelerates structural destruction of the joints, thus improving healthrelated quality of life [1-6]. For optimal effectiveness, adalimumab is usually given in combination with methotrexate (MTX) [7].

Here, we present results of an ongoing noninterventional study in which patients with active RA received 12 months of adalimumab therapy. The objectives of this study were to evaluate the effectiveness of adalimumab in routine clinical practice and to explore the potential impact of patient and disease characteristics in response to adalimumab therapy.

\section{Patients and methods}

\section{Study design}

This report utilizes data from a single-arm, multicenter, prospective, observational, noninterventional study of RA patients starting adalimumab therapy at their clinician's decision during routine clinical practice at 374 rheumatology centers and clinical practices in Germany. After a baseline visit (prior to initiation of adalimumab therapy), follow-ups were scheduled at months 3, 6 and 12 during the first year of adalimumab treatment.

In this report, we present results for up to 12 months of adalimumab therapy including all eligible patients who enrolled between April 2003 and March 2009. The primary objectives of this study were to evaluate the effectiveness of adalimumab in RA patients during routine clinical practice and to explore the potential impact of patient and disease characteristics in response to adalimumab therapy.

Patients in the adalimumab noninterventional study were required to: (1) be at least 18 years of age with active RA; (2) have a clinical indication for treatment with a TNF- $\alpha$ inhibitor and no contraindications; (3) have not been given adalimumab previously; and (4) provide written consent for participation. Patients who had received prior treatment with TNF- $\alpha$ inhibitors other than adalimumab were eligible for study inclusion. All patients were informed about the objectives of the study and gave written consent for their voluntary participation and the anonymous use of their personal data in statistical analyses. Because of the noninterventional, observational nature of this study, ethics approval was not required by German law.
All patients who had baseline documentation within 14 days of starting therapy, baseline DAS28 $\geq 3.2$, and no previous adalimumab therapy were eligible for study inclusion (data analysis set). Because a primary goal of this study was to evaluate factors that affected therapeutic response at 12 months, patients included in the regression analysis set were required to have data documented for the DAS28 and FFbH at baseline and at month 12 of follow-up. In addition, patients with poor baseline documentation were excluded.

RA treatment was performed according to routine clinical practice, with adalimumab given subcutaneously at the recommended dose of $40 \mathrm{mg}$ every other week according to label. Concurrent treatment with MTX was recommended according to the German Summary of Product Characteristics unless contraindicated.

\section{Assessments of effectiveness}

RA disease activity and functional capacity in daily activities were selected as target variables of effectiveness. Disease activity was assessed by the disease activity score-28 joints (DAS28). The DAS28 varies between 0 and 10, with 10 indicating the highest degree of disease activity [7, 8].

The Funktionsfragebogen Hannover $(\mathrm{FFbH})$ questionnaire was used to assess patient function. The $\mathrm{FFbH}$ is a self-administered patient questionnaire comprising 18 questions on functional capacity in activities of daily living and is comparable to the health assessment questionnairedisability index (HAQ-DI) [9]. The numerically coded responses to the questions are combined to provide a total score that indicates the degree of remaining functional capacity (with $0 \%$ indicating maximal impairment and $100 \%$ maximal functional capacity) [10].

Month 12 values for DAS28 and FFbH were used as endpoints for effectiveness.

\section{Data analysis}

Descriptive statistics or frequencies were computed for all data as appropriate. Due to the documentary nature of this noninterventional study, data were not available for all assessments, and thus the sample size differed among parameters for each visit. All patients in the regression analysis set had baseline and 12 month data for the main outcome parameters (DAS28 and FFbH). For the data analysis set, supportive analyses using the last observation carried forward (LOCF) method were conducted to determine DAS28 and FFbH outcomes. LOCF analyses utilized the last available data from all patients with a documented withdrawal at or before month 12 .

In contrast to randomized clinical trials, data collected in a noninterventional observational study are not controlled 
for potentially treatment-confounding parameters such as patient and disease characteristics. Since all RA patients with the indication for adalimumab treatment were eligible for our study, our cohort of patients was very heterogeneous in terms of disease and patient characteristics such as disease duration and activity, comorbidities, concomitant medication, and treatment history. Regression analysis is the statistical method of choice to identify the relevant treatment-modifying variables and to quantify their impact on therapy outcome $[11,12]$.

Multiple regression analyses were utilized to evaluate two different endpoints: change in DAS28 from baseline to month 12 and change in FFbH from baseline to month 12 . For each of the two measures of therapeutic response, a regression model was built to identify variables that potentially modified the therapeutic effect. All 35 patients and disease characteristics determined at baseline prior to treatment with adalimumab were entered into each model using a stepwise (forward selection and backward elimination) technique. Only those variables (predictors) showing an independent, statistically significant contribution to the respective endpoint (DAS28 or $\mathrm{FFbH}$ ) at month 12 were retained in the regression model for that endpoint. The coefficient of determination $r^{2}$ and the beta weights indicate the gain in explanatory power and the strength of influence, respectively [13].

Based on the results of the regression analyses, exploratory subgroup analyses employing mean comparisons were performed on relevant and statistically significant parameters to determine how these characteristics were associated with therapeutic outcome.

\section{Results}

Baseline patient characteristics

The data analysis set consisted of 3,404 patients. Baseline demographic and disease characteristics are presented in Table 1. Patients had a mean age of 54.6 years, were mostly (78\%) women, exhibited moderate to high disease activity (mean DAS28 of 5.9, range 3.4-8.3), and had a mean disease duration of 11.7 years. More than $90 \%$ of patients received a diagnosis of RA more than 2 years before study enrollment, and almost half of the patients received the diagnosis more than 10 years ago.

Almost all (95.7\%) patients had received prior therapy with at least one conventional DMARD, mostly MTX $(83.0 \%)$. Approximately, $30 \%$ of the patients had been previously treated with at least one biologic agent and $6.8 \%$ had received prior treatment with two or more biologic agents. The most frequently used biologic agents were etanercept $(20.7 \%)$ and infliximab (13.4\%). Mean treatment
Table 1 Baseline characteristics of patients at the time of initiation of adalimumab therapy

\begin{tabular}{|c|c|c|}
\hline Parameter & $\begin{array}{l}\text { Data analysis } \\
\text { set } N=3,404\end{array}$ & $\begin{array}{l}\text { Regression } \\
\text { analysis set } \\
N=2,625\end{array}$ \\
\hline Age, years (SD) & $54.6(12.7)$ & $54.6(12.7)$ \\
\hline Females, \% & 77.7 & 77.4 \\
\hline BMI, $\mathrm{kg} / \mathrm{m}^{2}(\mathrm{SD})$ & $26.0(4.8)$ & $25.9(4.7)$ \\
\hline Disease duration, years (SD) & $11.7(9.2)$ & $11.7(9.1)$ \\
\hline Tender joint count (SD) & $12.4(7.2)$ & $12.6(7.2)$ \\
\hline Swollen joint count (SD) & $9.6(6.2)$ & $9.7(6.2)$ \\
\hline $\mathrm{CRP}, \mathrm{mg} / \mathrm{L}$ (SD) & $33.3(62.7)$ & $33.4(63.5)$ \\
\hline $\mathrm{ESR}, \mathrm{mm} / \mathrm{h}(\mathrm{SD})$ & $34.2(23.0)$ & $34.1(22.7)$ \\
\hline Rheumatoid factor & 77.7 & 78.2 \\
\hline Erosive changes, $\%$ & 77.1 & 77.5 \\
\hline Rheumatoid nodules, $\%$ & 26.0 & 26.0 \\
\hline Joint replacement surgery, $\%$ & 16.9 & 16.6 \\
\hline Patient global assessment-VAS (SD) & $6.5(1.8)$ & $6.5(1.8)$ \\
\hline DAS28 (SD) & $5.9(1.2)$ & $5.9(1.1)$ \\
\hline $\begin{array}{l}\mathrm{FFbH}, \% \text { remaining } \\
\quad \text { functional capacity }(\mathrm{SD})\end{array}$ & $58.3(23.1)$ & $59.0(23.1)$ \\
\hline \multicolumn{3}{|l|}{ Concomitant therapy } \\
\hline Any conventional DMARD, $\%$ & 74.5 & 76.1 \\
\hline MTX, \% & 54.2 & 56.4 \\
\hline Leflunomide, $\%$ & 21.1 & 20.4 \\
\hline Analgesics, $\%$ & 26.2 & 25.5 \\
\hline NSAIDs (nonspecific), $\%$ & 45.0 & 45.8 \\
\hline COX-2 inhibitors, $\%$ & 20.7 & 21.2 \\
\hline Glucocorticoids, $\%$ & 86.0 & 85.6 \\
\hline
\end{tabular}

Data are presented as mean values (standard deviation [SD]) unless otherwise indicated

$B M I$ body mass index, $C O X-2$ cyclo-oxygenase 2, CRP C-reactive protein, DAS28 Disease Activity Score- 28 joints, DMARD diseasemodifying antirheumatic drug, ESR erythrocyte sedimentation rate, FFbH Funktionsfragebogen Hannover functional capacity questionnaire, NSAIDs nonsteroidal anti-inflammatory drugs, VAS visual analog scale

duration for prior biologic agents ranged from a mean of 12.6 months for anakinra to 15.3 months for infliximab. The most frequent reasons for discontinuation of biologic agents were lack of effectiveness $(66.1 \%)$ and lack of tolerance $(24.7 \%)$.

Regression analysis set

The regression analysis set consisted of patients with data documented for the DAS2 8 and FFbH at baseline and at month 12 of follow-up. A total of 779 patients withdrew from the study at month 3 or month 6 ; these patients did not have data at month 12 and therefore did not meet the 
criteria for inclusion in the regression analysis set $(N=$ 2,625). An additional 233 patients withdrew at 12 months. Because complete data for month 12 were available for these patients, they were included among the 2,625 patients in the regression analysis set. The most frequent reasons for withdrawal in the first 12 months of therapy were lack of efficacy (538 patients) and adverse drug reactions (247 patients).

Baseline characteristics for the data analysis set and regression analysis set were similar (Table 1).

Impact of treatment on concomitant therapy

The proportions of patients receiving concomitant therapy at the time of initiation of adalimumab therapy were similar in the data analysis set and the regression analysis set (Table 1). At the time of adalimumab treatment initiation, approximately $75 \%$ of patients were receiving one or more conventional DMARDs, mostly MTX or leflunomide. In the regression analysis set, the percentage of patients with concomitant DMARD therapy decreased to $69.7 \%$ after 12 months of treatment with adalimumab. However, the percentage of patients receiving concomitant treatment with MTX (as recommended) remained stable at $54.7 \%$. The percentage of patients taking leflunomide was reduced by about one third during the same time interval (from 20.4 to $13.9 \%$ ).

In the regression analysis set, the percentage of patients receiving glucocorticoids in addition to adalimumab decreased from $85.6 \%$ at baseline to $73.9 \%$ at month 12 , and the mean equivalent dose of prednisolone decreased from 8.8 to $5.8 \mathrm{mg} /$ day in the same time interval. The proportion of patients with concomitant painkillers was reduced for nonsteroidal anti-inflammatory drugs (NSAIDs; from 45.8 to $37.4 \%$ ), cyclooxygenase-2 (COX-2) inhibitors (from 21.2 to $13.3 \%$ ), and analgesics (from 25.5 to $16.6 \%$ ).

Impact of treatment on disease activity and functional capacity

In the regression analysis set, disease activity decreased (from a mean DAS28 score of 5.9-3.9) and functional activity improved (from a mean $\mathrm{FFbH}$ score of 59.0-68.4\% points; higher $\mathrm{FFbH}$ scores indicate greater functional capacity) during the first 12 months of adalimumab treatment (Fig. 1; Table 2). Outcomes were similar when the 1,012 patients with documented withdrawals were included in LOCF analyses of the data analysis set $(N=3,404$; Table 2). Mean DAS28 scores decreased from 5.9 to 4.2 at month 12 in the data analysis set and mean $\mathrm{FFbH}$ scores increased from 58.3 to $65.7 \%$ points. However, compared to the regression analysis set, mean DAS28 scores in the

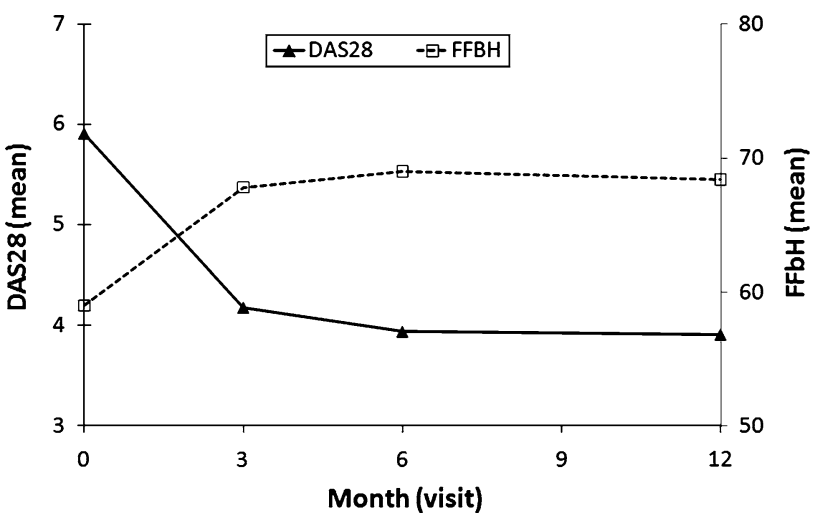

Fig. 1 Therapeutic response to adalimumab in patients in the regression analysis set $(N=2,625)$

data analysis set were slightly higher at month 12 (4.2 vs. 3.9) and mean FFbH scores were slightly lower (65.7 vs. $68.4 \%$ points), consistent with the observation that lack of efficacy accounted for the largest proportion of withdrawals.

The greatest improvements in disease activity and functional capacity occurred during the first 3 months of adalimumab therapy (Fig. 1). Mean DAS28 and FFbH scores continued to improve at 6 months and were sustained throughout 12 months. The DAS28 and FFbH scores displayed a low correlation throughout the course of the study ( $r=-0.41$ at baseline and -0.5 at month 12 ).

At 12 months, $19.1 \%$ of patients in the regression analysis set were in clinical remission, defined as DAS28< 2.6 points [14], $41.9 \%$ of patients had experienced a clinically relevant functional improvement, defined as an individual $\mathrm{FFbH}$ increase of 11 or more percentage points from baseline [15], and $57.8 \%$ were functionally independent (FFbH $\geq 67 \%$ points) [16] (Table 2). However, $9.1 \%$ of patients experienced a clinically significant decrease in functional capacity ( $\mathrm{FFbH}$ decrease of at least $11 \%$ points) during this time period. The proportions of patients with clinical improvements were slightly lower in the data analysis set (Table 2).

Patient and disease characteristics with an influence on therapeutic effectiveness

In the regression model for disease activity $(N=2,625), 8$ of the 35 tested variables showed a significant partial correlation with changes in DAS28 at month 12 and therefore can be regarded as having an impact on effectiveness in terms of disease activity (Table 3 ). Among these variables, baseline DAS28 exerted the strongest influence on therapeutic gain. High DAS28 baseline values correlated with reduced DAS28 values at month 12 and thus had a positive impact on reduction in disease activity. The additional 
Table 2 Disease activity (DAS28) and functional capacity $(\mathrm{FFbH})$ at baseline, month 3, and month 12 in the data analysis set $(N=3,404$, last observation carried forward) and the regression analysis set $(N=2,625)$

\begin{tabular}{|c|c|c|c|c|c|c|}
\hline \multirow[t]{3}{*}{ Outcome } & \multicolumn{6}{|c|}{ Month after inclusion (selection) } \\
\hline & \multicolumn{3}{|c|}{ Data analysis set $(N=3,404)$} & \multicolumn{3}{|c|}{ Regression analysis set $(N=2,625)^{*}$} \\
\hline & Baseline & Month 3 & Month 12 & Baseline & Month 3 & Month 12 \\
\hline \multicolumn{7}{|l|}{ DAS28 } \\
\hline Mean DAS28 (SD) & $5.9(1.2)$ & $4.5(1.5)$ & $4.2(1.5)$ & $5.9(1.1)$ & $4.2(1.4)$ & $3.9(1.4)$ \\
\hline$\%$ of patients in remission $(\mathrm{DAS} 28<2.6)$ & 0.0 & 11.4 & 16.2 & 0.0 & 14.1 & 19.1 \\
\hline \multicolumn{7}{|l|}{$\mathrm{FFbH}$} \\
\hline Mean FFbH percentage points (SD) & $58.3(23.1)$ & $65.1(23.5)$ & $65.7(24.1)$ & $59.0(23.1)$ & $67.8(22.7)$ & $68.4(23.5)$ \\
\hline \multicolumn{7}{|l|}{$\begin{array}{l}\% \text { of patients with clinically relevant } \\
\text { FFbH changes ( } \pm 11 \% \text { points }) \text { compared to baseline }\end{array}$} \\
\hline Clinically relevant improvement ( $\geq 11 \%$ points) & - & 32.4 & 36.8 & - & 38.3 & 41.9 \\
\hline Clinically relevant deterioration ( $\geq-11 \%$ points) & - & 6.8 & 11.0 & - & 5.6 & 9.1 \\
\hline No clinically relevant change ( $< \pm 11 \%$ points $)$ & - & 60.7 & 52.1 & - & 56.1 & 49.0 \\
\hline$\%$ of functionally independent patients & & & & & & \\
\hline (FFbH $\geq 67 \%$ points) & 38.4 & 52.3 & 52.8 & 39.5 & 57.0 & 57.8 \\
\hline
\end{tabular}

DAS28 Disease Activity Score-28 joints, FFbH Funktionsfragebogen Hannover functional capacity questionnaire, $S D$ standard deviation

* Month 3 regression analysis set data consisted of 2,421 patients for DAS28 analyses and 2,484 patients for FFbH analyses

effects of all other baseline parameters were less pronounced. Other variables with a positive effect on reduced disease activity were high $\mathrm{FFbH}$ scores at baseline, concomitant MTX therapy, and male gender. Previous biologic treatment, older age, increased number of tender joints, and use of nonselective NSAIDs were associated with reduced therapeutic gain as assessed by DAS28.

The 11 parameters that modified the increase in functional capacity at month 12 (Table 4) were only partially identical with the ones associated with reduced disease activity. The strongest influence was exerted by baseline $\mathrm{FFbH}$ scores; high functional capacity at baseline was associated with a less pronounced increase of $\mathrm{FFbH}$. Accordingly, patients with low functional capacity at baseline were more likely to achieve functional improvements during adalimumab treatment. Variables with less pronounced effects that were associated with reduced functional improvement were disease duration, previous joint replacement surgery, older age, previous biologic therapies, and high body mass index (BMI). Employment, male gender, use of COX-2 inhibitors, ESR levels, and baseline global patient assessment had a positive influence on increases in functional capacity.

No significant partial correlation with the two endpoints of therapeutic outcome was found for CRP levels, subjective pain experience, subjective limitations, number of concomitant DMARD therapies, duration of previous DMARD therapies, erosive changes, or concomitant diseases.
Accordingly, we conclude that these characteristics do not independently contribute to therapy success or failure.

Analysis of selected patient subgroups

Subgroup analysis was performed on selected significant parameters to illustrate how these patient and disease characteristics impact therapeutic outcome. When classified into patients with high or moderate/low disease activity (baseline DAS28 value $>5.1$ and $\leq 5.1$, respectively), the group of patients with high baseline DAS28 values experienced a larger reduction in mean disease activity (from a mean DAS28 of $6.4-4.2$ over 12 months) than the group with moderate/low baseline DAS28 (from a mean DAS28 of 4.4-3.2).

A similar pattern was seen for patient function. When patients were classified by baseline $\mathrm{FFbH}$ scores, patients with higher physical impairment (baseline $\mathrm{FFbH}$ score $\leq 50 \%$ points) improved from a mean of 34.2-50.9 points during 12 months of treatment with adalimumab, while those with less impairment (baseline $\mathrm{FFbH}$ score $>50 \%$ points) had much lower levels of improvement (from a mean of 73.9-78.9 points). Despite the greater extent of improvement, patients with high disease activity and low functional capacity at baseline did not reach the DAS28 and $\mathrm{FFbH}$ levels of the groups of patients with better baseline values.

Previous biologic treatment was associated with less pronounced therapeutic success of adalimumab for both 
Table 3 Parameters with significant partial correlation with DAS28 changes at month 12 in the regression analysis set

\begin{tabular}{|c|c|c|c|c|}
\hline \multirow[t]{2}{*}{ Regression model DAS28, $N=2,625$} & \multicolumn{4}{|l|}{ Model values } \\
\hline & & Constant & Total $R^{2}$ & $P$-value \\
\hline & & 1.524 & 0.2118 & $<0.001$ \\
\hline Variable & Value & Beta-weight & Partial $r^{2}$ & $P$-value \\
\hline \multicolumn{5}{|c|}{ Positive predictors for decrease in disease activity } \\
\hline Baseline DAS28 ${ }^{\mathrm{a}}$ & Continuous (0-10 units) & -0.707 & 0.156 & $<0.001$ \\
\hline Baseline $\mathrm{FFbH}^{\mathrm{b}}$ & Continuous (0-100\% points) & -0.006 & 0.008 & $<0.001$ \\
\hline Concomitant MTX & $1=$ yes, $0=$ no & -0.161 & 0.003 & 0.0013 \\
\hline Gender & $1=$ male, $0=$ female & -0.182 & 0.003 & 0.0027 \\
\hline \multicolumn{5}{|c|}{ Negative predictors for increase in disease activity } \\
\hline Previous biologics & Number $(0,1,2,3,4)$ & 0.314 & 0.020 & $<0.001$ \\
\hline Age (years) & Continuous & 0.012 & 0.014 & $<0.001$ \\
\hline Tender joint count & Number (0-28) & 0.024 & 0.006 & $<0.001$ \\
\hline NSAIDs (nonselective) & $1=$ yes, $0=$ no & 0.140 & 0.002 & 0.0056 \\
\hline
\end{tabular}

DAS28 Disease Activity Score-28 joints, FFbH Funktionsfragebogen Hannover functional capacity questionnaire, MTX methotrexate, NSAIDs nonsteroidal anti-inflammatory drugs

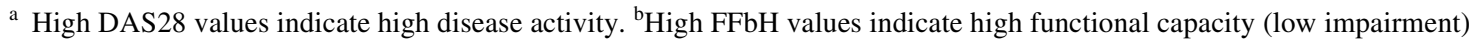

Table 4 Parameters with significant partial correlation with FFbH changes at month 12 in the regression analysis set

\begin{tabular}{|c|c|c|c|c|}
\hline \multirow[t]{2}{*}{ Regression model $\mathrm{FFbH}, N=2,625$} & \multicolumn{4}{|l|}{ Model values } \\
\hline & & Constant & Total $R^{2}$ & $P$-value \\
\hline & & 36.647 & 0.205 & $<0.001$ \\
\hline Variable & Value & Beta-weight & Partial $r^{2}$ & $P$-value \\
\hline \multicolumn{5}{|c|}{ Positive predictors for increase in functional capacity } \\
\hline Employment & $1=$ yes, $0=$ no & 3.078 & 0.023 & $<0.001$ \\
\hline Gender & $1=$ male, $0=$ female & 3.160 & 0.005 & $<0.001$ \\
\hline COX-2 inhibitors & $1=$ yes, $0=$ no & 2.892 & 0.005 & $<0.001$ \\
\hline ESR & Continuous & 0.047 & 0.004 & $<0.001$ \\
\hline Patient global assessment & Number $(0-10)$ & 0.653 & 0.003 & $<0.001$ \\
\hline \multicolumn{5}{|c|}{ Negative predictors for increase in functional capacity } \\
\hline Baseline $\mathrm{FFbH}^{\mathrm{a}}$ & Continuous (0-100\% points) & -0.317 & 0.126 & $<0.001$ \\
\hline Disease duration & Continuous & -0.171 & 0.017 & $<0.001$ \\
\hline Joint replacement & $1=$ yes, $0=$ no & -4.277 & 0.008 & $<0.001$ \\
\hline Age (years) & Continuous & -0.123 & 0.005 & $<0.001$ \\
\hline Previous biologics & Number $(0,1,2,3,4)$ & -2.149 & 0.005 & $<0.001$ \\
\hline $\operatorname{BMI}\left(\mathrm{kg} / \mathrm{m}^{2}\right)$ & Continuous & -0.258 & 0.005 & $<0.001$ \\
\hline
\end{tabular}

$B M I$ body mass index, $C O X-2$ cyclo-oxygenase 2, ESR erythrocyte sedimentation rate, FFbH Funktionsfragebogen Hannover functional capacity questionnaire, MTX methotrexate

${ }^{\text {a }}$ High $\mathrm{FFbH}$ values indicate high functional capacity (low impairment) 


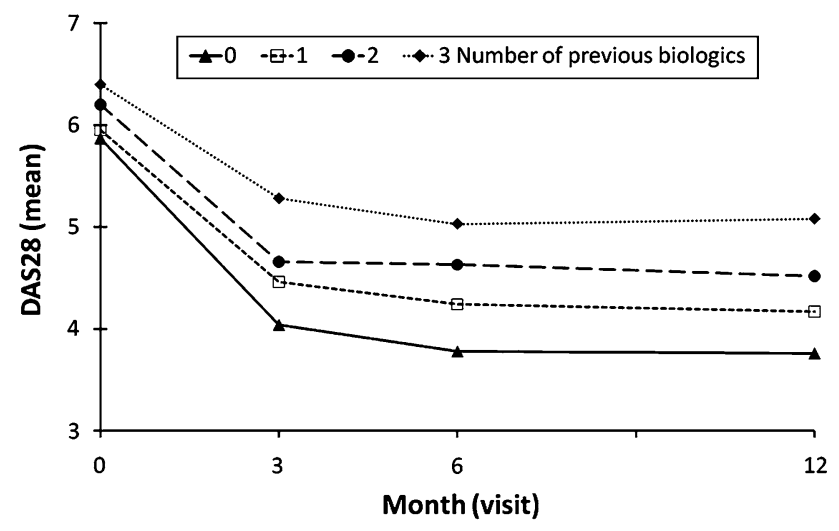

Fig. 2 DAS28 during adalimumab treatment by number of previous biologic therapies in the regression analysis set $(N=2,625)$. Patient numbers at month $0,3,6,12$. 0 previous biologic: $n=1,834,1,760$, $1,671,1,834$. 1 previous biologic: $n=623,560,557,623.2$ previous biologics: $n=154,141,140,154.3$ previous biologics: $n=14,14,12,14$

disease activity and functional capacity. When classified into subgroups by the number of previous biologic therapies, patients with 3 previous biologic therapies had higher DAS28 values at baseline compared with patients with 1 or 0 previous biologic therapies. While all subgroups showed a decrease in DAS28 values during the 12 months of adalimumab treatment, this decrease was most pronounced in patients without previous biologic treatment and least pronounced in those with 2 or 3 previous biologic therapies (Fig. 2). The effect of prior biologic therapy was also evident when analyzing functional capacity. Patients without previous biologic treatment showed the highest baseline FFbH values (60.9\% points) and improved to $70.7 \%$ points at month 12 . With increasing numbers of previous biologic therapies, $\mathrm{FFbH}$ baseline and month 12 scores declined steadily. Patients with 3 previous biologic therapies had a mean $\mathrm{FFbH}$ baseline value of $48.8 \%$ points and gained only $4.4 \%$ points (to $53.2 \%$ points) by month 12 .

\section{Discussion}

In this study, we evaluated outcomes of RA patients treated with adalimumab in routine clinical practice. During the 1 -year treatment period, adalimumab reduced disease activity and increased functional capacity (Fig. 1). These changes were evident at month 3 and were maintained throughout the 12-month observation period.

Compared to other adalimumab studies, this study is distinct with respect to its size and observational, noninterventional nature. Noninterventional observational studies and registries include large numbers of patients that are heterogeneous in terms of disease activity, disease duration, age, treatment history, and other patient and disease characteris- tics. The lack of specified entry criteria greatly expands the population of patients included in noninterventional studies. Zink et al. reported that only $27-28 \%$ of patients enrolled in the German biologics register were eligible for the major randomized adalimumab trials. Furthermore, response rates were lower among patients in the register who would not have been eligible for inclusion in randomiszd clinical trials [17]. Observational studies are thus important tools that allow knowledge concerning treatment effects to be transferred to conditions of routine clinical practice.

Because the evaluation of factors affecting treatment response at 12 months was a primary goal of the study, we focused primarily on patients who had DAS28 and FFbH data at baseline and 12 months. This approach has been successfully used in analyses of data from the German biologics register, RABBIT [11]. The treatment response observed in this study is generally comparable with those from other studies of adalimumab therapy in smaller RA patient populations [18-20].

Large numbers of patients are required for the identification of characteristics that modify disease progression and therapeutic response by post hoc multivariate regression analysis. This condition was met in our study, which included 2,625 patients with data at baseline and month 12 . High disease activity was correlated with the greatest decrease in DAS28 at month 12 (Table 3), and high functional capacity was a strong negative predictor of increased functional capacity during treatment (Table 4), indicating that baseline patient disease status has substantial effects in response to treatment. Male gender was a positive predictor for therapeutic response for both disease activity and functional capacity, whereas previous biologic therapy and older age were negative predictors of therapeutic response for both analyses. Other predictors of therapeutic response were unique to DAS28 changes, including TJC, concomitant MTX, and concomitant NSAIDs, or to FFbH changes, including employment, patient global assessment, and ESR. Baseline $\mathrm{FFbH}$ was selected in both models, but served as a positive predictor for DAS28 decreases and a negative predictor for increased functional capacity. Although some of these variables, particularly male gender, previous biologic therapy, and age, have been found to be significant predictors of response or remission in RA [21-23], TJC has not previously been associated with therapeutic response. The negative impact of tender joints on DAS28 scores at 12 months was an unexpected finding, as the TJC is a pivotal component of the DAS28 and high DAS28 baseline values were correlated with strong improvements in disease activity. This effect was not observed for swollen joints. The association between tender joint count and DAS28 changes requires further exploration. Another issue that deserves additional study is the impact of NSAID use on response. 
Two other studies have identified NSAID use as a predictor of a positive therapeutic response [21,22], whereas our findings suggest that NSAID use is associated with a negative therapeutic response as assessed by DAS28.

Subgroup analyses were used to further explore therapeutic response patterns. These analyses supported the observation that patients with high disease activity and low functional capacity benefitted substantially from adalimumab therapy. Despite the excellent clinical response observed in these patients, though, they did not achieve the same DAS28 and FFbH levels as patients with better values at baseline. The effect of previous biologic treatment on the therapeutic success of adalimumab was also supported by a subgroup analysis (Fig. 2); patients with 2 or 3 previous biologic therapies showed only small DAS28 reductions, despite the fact that these groups of patients had high baseline DAS28 values, a parameter associated with improved therapeutic response. This finding calls into question the strategy of continuous cycling among different anti-TNF treatments. In a study in which patients with inadequate response to TNF inhibitors were treated with tocilizumab, an interleukin-6 receptor inhibitor, patients receiving the highest dose of tocilizumab showed similar ACR20 response rates irrespective of the number of failed antiTNF- $\alpha$ therapies [24]. Therefore, switching to a biologic agent with a different mode of action may be a preferable option under certain conditions.

The positive impact of concomitant MTX treatment on therapeutic response to adalimumab has been demonstrated in clinical trials, mainly in those involving MTX-naïve patients with early RA [1, 2, 25], but has not been studied as thoroughly in patients with longstanding RA and multiple DMARD exposure. The association between concomitant MTX and reduced disease activity shown here provides an important argument in favor of MTX plus adalimumab combination therapy, even in patients with longstanding disease.

On the basis of these data, we conclude that adalimumab therapy is effective in reducing disease activity and improving function in "real-world" RA patients during routine clinical practice. Patients with high disease activity and low functional capacity are particularly likely to benefit from initiation of adalimumab, but those who are older or have been treated with multiple biologic agents may experience a lower degree of improvement.

Acknowledgments Funding for this study, data analysis, and manuscript support was provided by Abbott GmbH \& Co. KG, Wiesbaden, Germany. The authors wish to thank all physicians who contributed to this noninterventional observational study, among them: A Seifert, Berlin; R Sprekeler, Zeven; M Vollmer, Mönchengladbach; K Lüthke, Dresden; R Dockhorn, Weener; B Krummel-Lorenz, Frankfurt; H Kellner, Munich; P Hrdlicka, Chemnitz; M Zellner, Ahrensburg, F Schuch, Erlangen; J Georgi, Damp; K Zeribi, Damp; T Heidler,
Berlin; K Babinsky, Halle; J Walter, Rendsburg; H Sörensen, Berlin; HH Euler, Hamburg; G Lorenz, Chemnitz; G Neeck, Hohenfelde; L Meier, Hofheim; M Richter, Rostock; A Kapelle, Welzow. We thank Britta Eichhorn, M.A. and Sharon L. Cross, Ph.D. for providing medical writing services on behalf of Abbott GmbH \& Co. KG.

Conflict of interest S. Kleinert, H.-P. Tony, A. Krause, M. Feuchtenberger, S. Wassenberg, C. Richter, E. Röther, and W. Spieler received honoraria, speaker's fees, or compensation for consultancies from Abbott GmbH \& Co. KG, the sponsor of this study. H. Gnann is a paid consultant for Abbott. B.M. Wittig is an employee of Abbott.

Open Access This article is distributed under the terms of the Creative Commons Attribution Noncommercial License which permits any noncommercial use, distribution, and reproduction in any medium, provided the original author(s) and source are credited.

\section{References}

1. Breedveld FC, Weisman MH, Kavanaugh AF et al. (2006) The PREMIER study: A multicenter, randomized, double-blind clinical trial of combination therapy with adalimumab plus methotrexate versus methotrexate alone or adalimumab alone in patients with early, aggressive rheumatoid arthritis who had not had previous methotrexate treatment. Arthritis Rheum 54(1):26-37. doi: 10.1002/art.21519

2. Burmester GR, Mariette X, Montecucco C et al (2007) Adalimumab alone and in combination with disease-modifying antirheumatic drugs for the treatment of rheumatoid arthritis in clinical practice: the research in active rheumatoid arthritis (ReAct) trial. Ann Rheum Dis 66(6):732-739. doi:10.1136/ard.2006.066761

3. Chen YF, Jobanputra P, Barton P et al (2006) A systematic review of the effectiveness of adalimumab, etanercept and infliximab for the treatment of rheumatoid arthritis in adults and an economic evaluation of their cost-effectiveness. Health Technol Assess 2006 10(42):iii-iv, xi-xiii, 1-229

4. Furst DE, Schiff MH, Fleischmann RM et al (2003) Adalimumab, a fully human anti-tumor necrosis factor-alpha monoclonal antibody, and concomitant standard anti-rheumatic therapy for the treatment of rheumatoid arthri-tis: results of STAR (Safety Trial of Adalimumab in Rheumatoid Arthritis). J Rheumatol 30(12):25632571

5. Keystone EC, Kavanaugh AF, Sharp JT et al (2004) Radiographic, clinical, and functional outcomes of treatment with adalimumab (a human anti-tumor necrosis factor monoclonal antibody) in patients with active rheuma-toid arthritis receiving con-comitant methotrexate therapy: a randomized, placebo-controlled, 52week trial. Arthritis Rheum 2004; 50(5):1400-1411. doi:10.1002/art. 20217

6. Van de Putte LB, Atkins C, Malaise M et al (2004) Efficacy and safety of adalimumab as monotherapy in patients with rheumatoid arthritis for whom previous disease modifying antirheumatic drug treatment has failed. Ann Rheum Dis 63(5):508-516. doi:10.1136/ ard.2003.013052

7. Furst DE, Keystone EC, Fleischmann R et al (2010) Updated consensus statement on biological agents for the treatment of rheumatic diseases, 2009. Ann Rheum Dis 69(Suppl 1):i2-i29. doi:10.1136/ard.2009.123885

8. Vander Cruyssen B, Van Looy S, Wyns B et al (2005) DAS28 best reflects the physician's clinical judgment of response to infliximab therapy in rheumatoid patients: validation of the DAS28 score in patients under infliximab treatment. Arthritis Res Ther 7(5): R1063-R1071. doi:10.1186/ar1787 
9. Lautenschläger J, Mau W, Kohlmann T et al (1997) Vergleichende Evaluation einer deutschen Version des Health Assessment Questionnaires (HAQ) und des Funktionsfragebogens Hannover (FFbH) [Comparative evaluation of a German version of the Health Assessment Questionnaire (HAQ) and the Hannover Functional Status Questionnaire (HFSQ)]. Z Rheumatol 56(3):144-155

10. Raspe HH, Hagedorn U, Kohlmann T, Mattussek S (1990) Der Funktionsfrage-bogen Hannover $(\mathrm{FFbH})$ : Ein Instrument zur Funktionsdiagnostik bei polyartikulären Gelenkerkrankungen [The Hannover Functional Status Questionnaire (HFSQ): an instrument for functional diagnostic in polyarticular joint disease]. In: Siegrist J (ed) Wohn-ortnahe Be-treuung Rheumakranker. Ergebnisse sozialwissenschaftlicher Evaluation eines Modellversuchs [Care in the neighborhood for patients suffering from rheumatic diseases. Social scientific evaluation results of a pilot project]. Schattauer, Stuttgart, pp 164-182

11. Listing J, Strangfeld A, Rau R et al. (2006) Clinical and functional remission: even though biologics are superior to conventional DMARDs overall success rates remain low-results from RABBIT, the German biologics register. Arthritis Res Ther 8(3):R66. doi: 10.1186/ar1933

12. Woolson RF (1987) Multiple linear regression. In: Woolson RF (ed) Statistical methods for the analysis of biomedical data. Wiley, New York, pp 295-300

13. Bortz J (2005) Partialkorrelation und multiple Korrelation [Partial correlation and multiple regression]. In: Bortz J (ed) Statistik für Human- und Sozialwissenschaftler [Statistics for human and social scientists], 6th edn. Springer, Heidelberg, pp 439-481

14. Fransen J, Creemers MC, van Riel PL (2004) Remission in rheumatoid arthritis: agreement of the disease activity score (DAS28) with the ARA preliminary remission criteria. Rheumatology (Oxford) 43(10):1252-1255. doi:10.1093/rheumatology/keh297

15. Raspe HH, Kindel P, Vesterling K, Kohlmann T (1987) Die Entwicklung der Funktionskapazität und der Schmerzintensität von 81 CP-Patienten unter einer Behandlung mit Azulfidine RA oder Aurothioglucose. [Development of functional capacity and pain intensity in $81 \mathrm{CP}$ patients during treatment with azulfidiene RA or aurothioglucose]. Z Rheumatol 46(2):71-75

16. Westhoff G, Listing J, Zink A (2000) Loss of physical independence in rheumatoid arthritis: interview data from a representative sample of patients in rheumatologic care. Arthritis Care Res 13(1):11-22

17. Zink A, Strangfeld A, Schneider M et al (2006) Effectiveness of tumor necrosis factor inhibitors in rheumatoid arthritis in an obser- vational cohort study: comparison of patients according to their eligibility for major randomized clinical trials. Arthritis Rheum 54:3399-3407. doi:10.1002/art.22193

18. Kievit W, Adang EM, Fransen J et al (2008) The effectiveness and medication costs of three anti-tumour necrosis factor $\alpha$ agents in the treatment of rheumatoid arthritis from prospective clinical practice data. Ann Rheum Dis 67(9):1229-1234. doi:10.1136/ ard.2007.083675

19. Weinblatt ME, Keystone EC, Furst DE et al (2003) Adalimumab, a fully human anti-tumor necrosis factor alpha monoclonal antibody, for the treatment of rheumatoid arthritis in patients taking concomitant methotrexate: The ARMADA trial. Arthritis Rheum 48(1):35-45. doi:10.1002/art.10697

20. Burmester GR, Ferraccioli G, Flipo R-M et al (2008) Clinical remission and/or minimal disease activity in patients receiving adalimumab treatment in a multinational, open-label, twelve-week study. Arthritis Rheum 59:32-41. doi:10.1002/art.23247

21. Hyrich KL, Watson KD, Silman AJ, Symmons DP (2006) British society for rheumatology biologics register: predictors of response to anti-TNF- $\alpha$ therapy among patients with rheumatoid arthritis: results from the British society for rheumatology biologics register. Rheumatology 45(12):1558-1565. doi:10.1093/rheumatology/kel149

22. Kristensen EL, Kapetanovic MC, Gülfe A, Söderlin M, Saxne T, Geborek P (2008) Predictors of response to anti-TNF therapy according to ACR and EULAR criteria in patients with established RA: results from the South Swedish Arthritis Treatment Group Register. Rheumatology 47(4):495-499. doi:10.1093/rheumatology/ken002

23. Katchamart W, Johnson S, Lin L-JL, Phumethum V, Salliot C, Bombardier C (2010) Predictors for remission in rheumatoid arthritis patients: a systematic review. Arthritis Care Res 62(8):1128-1143. doi:10.1002/acr.20188

24. Emery P, Keystone E, Tony HP et al (2008) IL-6 receptor inhibition with tocilizumab improves treatment outcomes in patients with rheumatoid arthritis refractory to anti-tumour necrosis factor biologicals: results from a 24 -week multicentre randomised placebo-controlled trial. Ann Rheum Dis 67(11):1516-1523. doi:10.1136/ard.2008.092932

25. Emery P, Genovese MC, van Vollenhoven R, Sharp JT, Patra K, Sasso EH (2009) Less radiographic progression with adalimumab plus methotrexate versus methotrexate monotherapy across the spectrum of clinical response in early rheumatoid arthritis. J Rheumatol 36(7):1429-1441 